\title{
CD45-positive cells of haematopoietic origin enhance chondrogenic marker gene expression in rat marrow stromal cells
}

\author{
NAZISH AHMED ${ }^{1 *}$, BREDA VOGEL ${ }^{2,3^{*}}$, EVA ROHDE $^{4}$, DIRK STRUNK $^{4}$, \\ JOACHIM GRIFKA $^{1}$, MICHAELA B. SCHULZ ${ }^{3}$ and SUSANNE GRÄSSEL ${ }^{1}$
}

\author{
${ }^{1}$ Experimental Orthopaedics, Department of Orthopaedics, University of Regensburg, Kaiser Karl V Allee-3, \\ 93077 Bad Abbach; ${ }^{2}$ Pharmaceutical Technology, University of Regensburg, 93053 Regensburg, Germany; \\ ${ }^{3}$ Institute of Pharmaceutical Sciences, Pharmaceutical Technology, Karl-Franzens University; ${ }^{4}$ Division \\ of Hematology and Department of Transfusion Medicine, Medical University, 8010 Graz, Austria
}

Received March 7, 2006; Accepted April 10, 2006

\begin{abstract}
Adult mesenchymal stem cells (MSCs) can be readily isolated from bone marrow, expanded in culture and subsequently subjected to differentiation into various connective tissue lineages. In general, for animal studies separation of MSCs from other bone marrow-derived cells is achieved by sole adherence to plastic surface of tissue culture flasks; however, this procedure produces a heterogeneous cell population containing CD45-positive haematopoietic cells (HCs) and haematopoietic stem cells (HSCs). It is known, that mixed cell cultures consisting of cocultures of differentiated somatic cells with adult stem cells promote differentiation towards specific cell lineages. For determining the effect of the CD45-positive cell population on the differentiation potential of MSCs, we sorted out the bone marrow-derived adherent cells by immunomagnetic technique (MACS ${ }^{\mathrm{TM}}$ ) to attain a subpopulation of CD45-depleted cells. Herein, we show that the presence of adherent CD45-positive HCs not only promote expression of the chondrogenic marker genes Col2a1, COMP and Sox9, but also of Colla1, Colloal and to a certain degree Cbfal in MSCs when cultured in an appropriate three-dimensional environment. These observations constitute a step towards unravelling the influence of haematopoietic cells on chondrogenic differentiation of MSCs.
\end{abstract}

Correspondence to: Dr Susanne Grässel, Orthopädische Universitätsklinik Regensburg, Kaiser Karl V Allee-3, 93077 Bad Abbach, Germany

E-mail: susanne.graessel@klinik.uni-regensburg.de

${ }^{*}$ Contributed equally

Key words: rat marrow stromal cells, magnetic associated cell sorting, chondrogenic differentiation, haematopoietic cells, CD45

\section{Introduction}

Adult bone marrow stromal cells regarded as mesenchymal stem cells are progenitors of connective tissue cells, thus are excellent candidates for chondrogenic differentiation studies. The progeny of adult stem cells includes both daughter stem cells and committed progenitors with a more restricted differentiation potential. These progenitors in turn give rise to differentiated cell types. Mesenchymal stem cells (MSCs) can differentiate under specific cultural and physical conditions into multiple mesenchymal lineages namely, osteocytes, chondrocytes, adipocytes, astrocytes and myocytes $(1,2)$. Bone marrow consists of primarily non-adherent haematopoietic cells and haematopoietic stem cells $(99 \%)$ along with a minor population of MSCs $(\leq 1 \%)$. Both of the stem cell types are known to co-exist and have been suggested to cooperate in one another's differentiation (3). The role of MSCs in haematopoietic microenvironment formation is beginning to unravel, and it is assumed that the presence of adherent MSCs and their progeny facilitates HSC differentiation into granulocytes and erythrocytes both in vivo and in vitro (4-6). Conversely, in depth studies are scarce concerning the role of HSCs in the MSC commitment and differentiation towards a distinct lineage.

Adult rat MSCs are routinely isolated from tibio-femoral bone marrow by relying solely on their adherence to the plastic surface of tissue culture flasks $(7,8)$. The isolated cells constitute a heterogeneous population that always contains HCs; therefore, obtaining a pure population of non-haematopoietic cells remains difficult. This haematopoietic cell fraction varies depending on the species; being relatively high in initial cultures of mouse marrow cells (9) and $<30 \%$ in human cell cultures. In rat marrow stromal cells this trend has not been studied thoroughly, although the presence of haematopoietic cells in primary (7 days) and secondary (18 days) bone marrow cultures has been indicated (10). Human and murine MSC studies show subsequent loss of haematopoietic cell surface markers when cultures are maintained for 2 or 3 weeks. Prior to differentiation experiments the haematopoietic cell 
population is often separated from MSCs by employing a magnetic associated cell sorting (MACS) system. The system immunologically separates different cell types by labelling cell surface antigens with magnetic beads followed by sorting through a magnetic column. As a unique identifying marker for MSCs is still lacking the negative selection protocol is carried out using CD34 (My10) or CD45 (leukocyte common antigen) for exclusion of undesired positively labelled haematopoietic cells.

One of the major differentiation pathways of MSCs occurs along the chondrogenic lineage which can occur autonomously in three separate mesenchymal cell lines, cranial neural crest, sclerotome cells and lateral plate mesoderm cells (11). The first stage of chondrogenic differentiation is conversion of undifferentiated MSCs to committed osteochondroprogenitor cells leading to cell condensation and growth arrest. The major proteins involved in condensation initiation are either fibronectin or belong to the transforming growth factor $\beta$ family of proteins (TGFß-1, -2 and -3). Cells present at the centre of the condensation nodules first form pre-chondrocytes and then chondrocytes which start to produce cartilage matrix marked by upregulation of structural protein genes such as Col2a1, Col9a2, Coll1a2, aggrecan and COMP. Expression of chondrocyte marker genes is controlled by members of the Sox-family, in particular Sox 9 has been characterized as a master transcription factor with essential direct or indirect regulatory effects exerted along the entire chondrogenic differentiation pathway. It is expressed in all chondroprogenitor cells and also in differentiated chondrocytes (12-15).

In this study, we have evaluated the degree of haematopoietic cell coexistence in primary cultures of rat MSCs and their influence on the chondrogenic differentiation potential of MSCs. We have determined the difference in chondrogenic marker gene expression of heterogeneous MSCs isolated by sole adherence to plastic surfaces compared to that of a more homogeneous subpopulation of MACS-sorted CD45depleted cells.

\section{Materials and methods}

MSC isolation and primary cell culture. A modified version of the MSC isolation procedure from rat bone marrow was optimized (16,17). Briefly, 6-week-old male Sprague-Dawley rats were sacrificed with $\mathrm{CO}_{2}$ and tibiae and femora were aseptically removed. The bones were cut from the middle and centrifuged in a $1.5 \mathrm{ml}$ tube at $2000 \mathrm{rpm}$ for $3 \mathrm{~min}$ (mini centrifuge-Eppendorf). The centrifuged bone marrow cells were collected and homogenized with 18,21 and $23 \mathrm{~g}$ needles and seeded at the rate of $2.5 \times 10^{5}$ cells $/ \mathrm{cm}^{2}$ in $175 \mathrm{~cm}^{2}$ tissue culture flasks in proliferation medium containing $5 \%$ glutamate, $1 \%$ antibiotics/antimycotics and 10\% FBS (Gibco, Invitrogen, UK; Lot No.: 40F7430K) in $\alpha$-MEM (Sigma Aldrich, Germany). All non-adherent cells were removed on the 3rd day and the adherent cells were proliferated.

Magnetic separation of cells (MACS). When the flasks were $\sim 75 \%$ confluent (9-11 days) cells were harvested with trypsin digestion. Separation procedure was carried out according to the manufacturer's instructions; in short, cells were incubated with $4 \mu 1$ of CD45 antibody $/ 10^{6}$ cells for $5 \mathrm{~min}$ at $37^{\circ} \mathrm{C}$ followed by washing and a second incubation with goat antimouse secondary antibody coupled with magnetic beads (Miltenyi Biotech, Germany) for $15 \mathrm{~min}$ at $4^{\circ} \mathrm{C}$. The suspension was passed through a magnetic column (Miltenyi Biotec's LS-MACS columns); while labelled cells coupled to the magnetic field, the flow through containing unlabelled CD45-negative fraction was collected.

Immunofluorescence. Twenty thousand cells/chamber were seeded on chamber slides (Biocoat slides, BD, Bioscience) and incubated overnight at $37^{\circ} \mathrm{C}$ and $5 \% \mathrm{CO}_{2}$. Cells were blocked for $1 \mathrm{~h}$ at $37^{\circ} \mathrm{C}$ in Complete Mini 1:5 (Roche, Germany) plus 5\% normal goat serum and 1\% BSA in PBS. After washing with PBS, cells were stained with monoclonal antibodies directed against CD45 (Cb1 1502, Chemicon, Germany), CD68 and D7Fib (Acris, Germany) in 1:50 dilution ratio overnight at $4{ }^{\circ} \mathrm{C}$. The appropriate Alexa568 or Alexa488 conjugated secondary antibodies (goat anti-mouse, $5 \mu \mathrm{g} / \mathrm{ml}$; Molecular Probes, USA) were added for $1 \mathrm{~h}$ at RT. After washing, slides were permanently mounted with Dako fluorescent mounting medium (Dako, USA) and covered with coverslips. Slides were evaluated with scanning laser microscopy (C1 confocal microscope from Nikon) and photos were taken with a Nikon C4 camera and software.

Flow cytometric analysis. Cells obtained from one animal $\left(\sim 8 \times 10^{7}\right.$ cells, as determined after $\mathrm{NH}_{4} \mathrm{Cl}$ lysis of the erythrocytes) were seeded in $4 \times 150 \mathrm{~cm}^{2}$ flasks $\left(5 \times 10^{5}\right.$ cells $\left./ \mathrm{cm}^{2}\right)$. The cells were left to adhere $1,2,3$ or 4 days before non-adherent cells were removed. Cells were trypsinized on day 7 after the isolation and resuspended in PBS. The cell suspension was first blocked for $15 \mathrm{~min}$ at $4^{\circ} \mathrm{C}$ with sheep serum followed by incubation with antibodies against rat CD45-PE (mouse IgG1 against rat CD45, Acris Antibodies, Germany), CD29-FITC (Hamster IgM against rat CD29, BD Pharmingen, USA) and with corresponding isotype controls for $30 \mathrm{~min}$ at $4^{\circ} \mathrm{C}$. Cells were washed with PBS containing sodium azide and bovine serum albumin. Shortly before the FACS, analysis propidium iodide was added to each sample in order to discriminate the dead cells which were excluded from the evaluation. All measurements were performed on a FACS-Calibur ${ }^{\circledR}$ instrument (Becton Dickenson, USA) with CellQuest data acquisition software (Becton Dickenson, USA). Data analysis was preformed with WinMDI 2.8 software (free access on http://facs.scripps.edu/software.html). A minimum of $1 \times 10^{4}$ viable cells was acquired per data set.

Chondrogenic differentiation. A batch of adherent cells (undepleted) was subjected directly to chondrogenesis favourable condition; the other batch was depleted of CD45positive cells by MACS prior to chondrogenic differentiation experiments. For chondrogenic differentiation, high density 3 -D cultures were attained by preparing cell-alginate amalgam in $1.2 \%$ alginate at a concentration of $10^{7}$ cells $/ \mathrm{ml}$. Cellalginate beads $(2-3 \mathrm{~mm})$ containing $\sim 10^{5}$ cells/bead, were formed by dropping the suspension into $102 \mathrm{mM} \mathrm{CaCl}_{2}$ solution. The culture was carried out in 12-well tissue culture plates for 4 weeks; medium was changed every 2-3 days. Ten alginate beads were removed every 7 th day for RNA isolation 


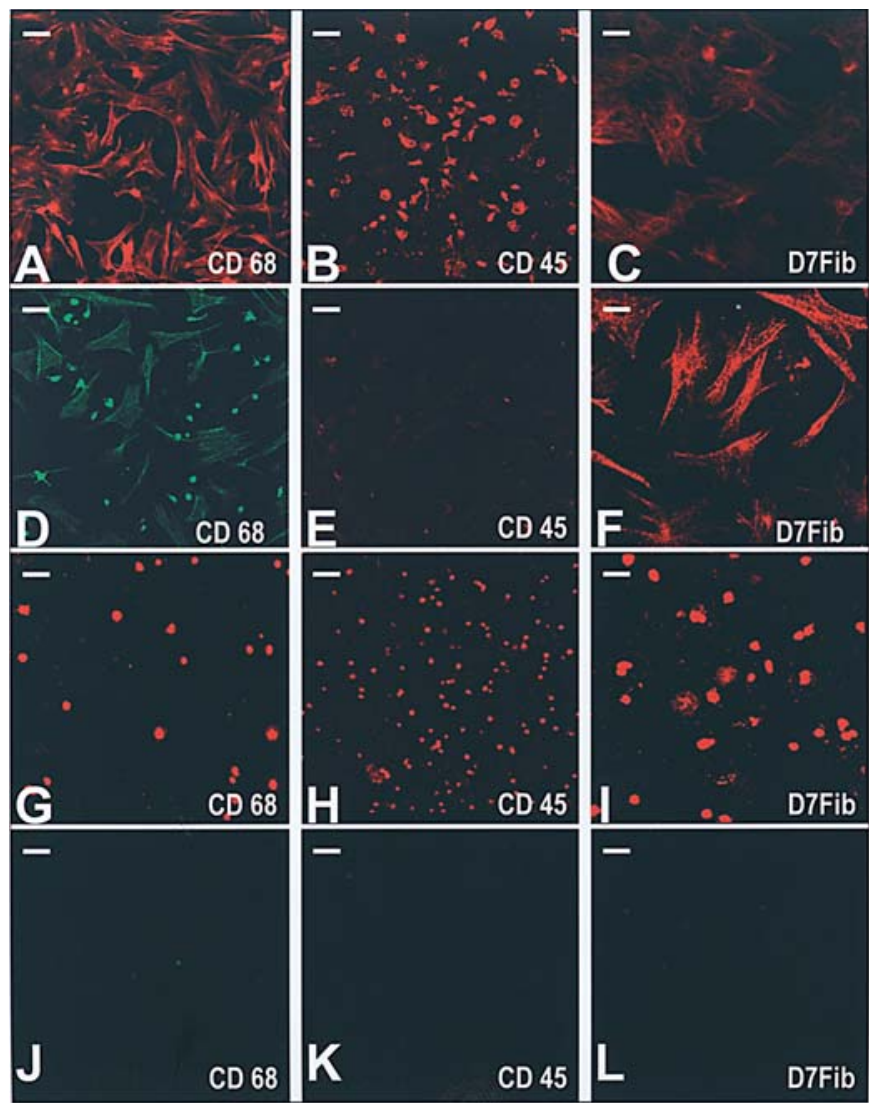

Figure 1. Morphological characterization of MSCs. Immunofluorescence imaging of heterogeneous adherent MSCs (A-C) and CD45-depleted MACS sorted cells (D-F), showing typical fibroblast-like spindle shaped cells, while CD45-positive cells (G-I) are small and rounded in appearance. The negative controls for all three antibodies are unstained (J-L). All three cell types are CD68- and D7fib-positive while the CD45-depleted cell population does not exhibit CD45 harbouring cells (E). Magnification is x200 for all panels, bars are $10 \mu \mathrm{m}$.

and gene expression analysis. To release the cells from alginate, the beads were incubated for $30 \mathrm{~min}$ at $37^{\circ} \mathrm{C}$ on a shaker in $55 \mathrm{mM}$ sodium citrate and $0.15 \mathrm{M}$ sodium chloride buffer; cells were recovered after a $3 \mathrm{~min}$ spin at $750 \mathrm{x} \mathrm{g}$. The chondrogenic medium contained; ITS+ premix $(6.25 \mu \mathrm{g} / \mathrm{ml}$ insulin, $6.25 \mathrm{ng} / \mathrm{ml}$ selenium acid, $6.25 \mu \mathrm{g} / \mathrm{ml}$ transferrin, $1.25 \mathrm{mg} / \mathrm{ml} \mathrm{BSA}$ and $5.35 \mu \mathrm{g} / \mathrm{ml}$ linoleic acid; BD Biosciences, USA), $110 \mu \mathrm{g} / \mathrm{ml}$ pyruvate, $40 \mu \mathrm{g} / \mathrm{ml}$ proline, $0.1 \mu \mathrm{M}$ dexamethasone, $50 \mu \mathrm{g} / \mathrm{ml}$ ascorbic acid and $10 \mathrm{ng} / \mathrm{ml} \mathrm{TGFß-3}$ (18) (R\&D Systems) in $\alpha$-MEM high glucose (Gibco, Invitrogen, UK). Alginate sodium salts were acquired from Sigma Aldrich, Germany (Cat. No.: A0682-100G).

RNA isolation and reverse transcription. RNA was isolated by an affinity column chromatography method with Ambion's RNAqueous4-PCR kit according to the manufacturer's protocol. For removal of possible DNA contamination, isolated RNA was incubated for $1 \mathrm{~h}$ at $37^{\circ} \mathrm{C}$ in 2 units of DNaseI enzyme (DNA-free, Ambion). RNA integrity was determined on $0.8 \%$ agarose-formaldehyde gels. The RiboGreen RNA quantification kit (Molecular Probes, USA) was used for determination of RNA concentration at $585 \mathrm{~nm}$ wavelength. For RNA conversion to cDNA, Invitrogen SuperScript II reverse transcriptase kit was used with $0.5-1 \mu \mathrm{g}$ of RNA in
$20 \mu 1$ of total reaction volume in presence of 40 units $/ \mu 1$ recombinant ribonuclease inhibitor (RNase OUT ${ }^{\circledR}$ ). Reverse transcription was carried out with $500 \mu \mathrm{g} / \mathrm{ml}$ of Oligo-dT primers, $10 \mathrm{mM}$ dNTPs and 200 units of SuperScript II enzyme in First-Strand buffer and 0.1 M DTT for $50 \mathrm{~min}$ at $42^{\circ} \mathrm{C}$ followed by an extension period of $15 \mathrm{~min}$ at $70^{\circ} \mathrm{C}$.

Relative quantitative PCR. Relative quantitative PCR was carried out with SYBR Green Dye I on ABI 7000 Prism Sequence detection system (Applied Biosystems, USA) according to the manufacturer's instructions. Briefly, $1 \mu 1$ of cDNA was amplified in $50 \mu \mathrm{l}$ final volume of $0.2 \mu \mathrm{M}$ of each primer suspended in SYBR green master mix (Applied Biosystems). Amplification parameters were the same for all primer pairs and were repeated for 40 cycles, denaturation at $95^{\circ} \mathrm{C}$ for $0.15 \mathrm{~min}$ and annealing at $60^{\circ} \mathrm{C}$ for $1 \mathrm{~min}$. Mean relative quantification (RQ) value evaluated from three independent experiments was plotted on a semi-logarithmic graph. RQ values were calculated by the software 'RQ study application v1.1' (ABI Prism 7000 SDS software v1.1) according to the $\Delta \Delta \mathrm{Ct}$ method using $B$-actin as endogenous control and undifferentiated MSCs (day 0) as calibrator. Primers were designed with either Primer3 (http://frodo.wi. mit.edu/cgi-bin/primer3/primer3_www.cgi) or 'Primer Express' software supplied by Applied Biosystems. All primers were manufactured at MWG - Biotech and are listed in Table I. Prior to RQ studies, a CT value (cycle threshold) standard curve with 10-fold diluted cDNA was plotted to determine PCR efficiency of each primer pair, only 90-100\% efficient primers were used. The efficiency was determined with $10^{(1 /-S)}-1$, where $S$ is the slope of the curve (19).

Statistical analysis. One way ANOVA and Student's t-test were employed for quantitative PCR experiments performed in triplicate. Results obtained by flow cytometry are the average of 4 independent experiments \pm standard error of mean. The level of significance was determined by Tukey's test with 2 group comparison.

\section{Results}

MSC isolation procedure and CD expression profile. To determine the influence of adherence time of rat bone marrow cells on the presence of CD45-positive haematopoietic cells, the cells were left to adhere for 3, 4 and 5 days before nonadherent and weakly attached cells were removed. Upon immunofluorescence analysis, the adherent cells showed a $\mathrm{D} \mathrm{fib}^{+}, \mathrm{CD} 8^{+}$and $\mathrm{CD} 45^{+/ \text {low }}$ expression profile regardless of the time of removal of non-adherent cells. Fig. 1 shows representative immunomicrographs of the cells left to adhere for 3 days and compares the staining of non-sorted and MACS-sorted cells after the first passage. Non-sorted cells, left for 3 days to adhere before medium removal, are contaminated with CD45-positive cells (Fig. 1A-C). After magnetic sorting, most of the CD45-positive cells were excluded, leaving a CD45-depleted cell fraction (Fig. 1D-F). However, we still observe few rounded cells among the CD68 positively stained fraction (Fig. 1D) of unknown surface antigen characterization. The CD45-positive cell fraction includes cells which stain for CD68 and D7Fib (Fig. 1G-I). 
Table I. Primers used for quantitative PCR.

\begin{tabular}{|c|c|c|}
\hline Gene/RefSeq \# & Amplicon (bp) & Primer $\left(5^{\prime}-3^{\prime}\right)$ \\
\hline $\begin{array}{l}\beta \text {-actin } \\
\text { NM_031144 }\end{array}$ & 104 & $\begin{array}{l}\text { Forward -GTAGCCATCCAGGCTGTGTT-3' } \\
\text { Reverse -CTGTAGGTGAATCGACTGTTGC-3' }\end{array}$ \\
\hline $\begin{array}{l}\text { Collal } \\
\text { RGD61817a }\end{array}$ & 59 & $\begin{array}{l}\text { Forward -TCCAGGGCTCCAACGAGA-3' } \\
\text { Reverse -CTGTAGGTGAATCGACTGTTGC-3' }\end{array}$ \\
\hline $\begin{array}{l}\text { Col2al } \\
\text { NM_012929 }\end{array}$ & 60 & $\begin{array}{l}\text { Forward -CCCCTGCAGTACATGCGG-3' } \\
\text { Reverse -CTCGACGTCATGCTGTCTCAAG-3' }\end{array}$ \\
\hline $\begin{array}{l}\text { Colloal } \\
\text { AJ131848 }\end{array}$ & 247 & $\begin{array}{l}\text { Forward -CCC TAT TGG ACC ACC AGG TA -3' } \\
\text { Reverse -TCT CTG TCC GCT CTT TGT GA -3' }\end{array}$ \\
\hline $\begin{array}{l}\text { COMP } \\
\text { NM_012834 }\end{array}$ & 167 & $\begin{array}{l}\text { Forward -TGACTTCGATGCTGACAAGG-3' } \\
\text { Reverse -GAACGATCTCCATTCCCTGA-3' }\end{array}$ \\
\hline $\begin{array}{l}\text { Sox9 } \\
\text { XM_343981 }\end{array}$ & 140 & $\begin{array}{l}\text { Forward -CTGAAGGGCTACGACTGGAC-3' } \\
\text { Reverse -TACTGGTCTGCCAGCTTCCT-3' }\end{array}$ \\
\hline $\begin{array}{l}\text { Cbfal } \\
\text { XM_34016 }\end{array}$ & 86 & $\begin{array}{l}\text { Forward -GGCCGGGAATGATGAGAACTA-3' } \\
\text { Reverse -AGATCGTTGAACCTGGCCACT-3' }\end{array}$ \\
\hline
\end{tabular}

${ }^{\mathrm{a}}$ Locus ID.

A

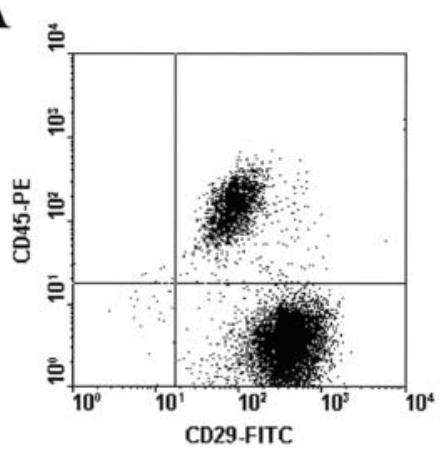

B

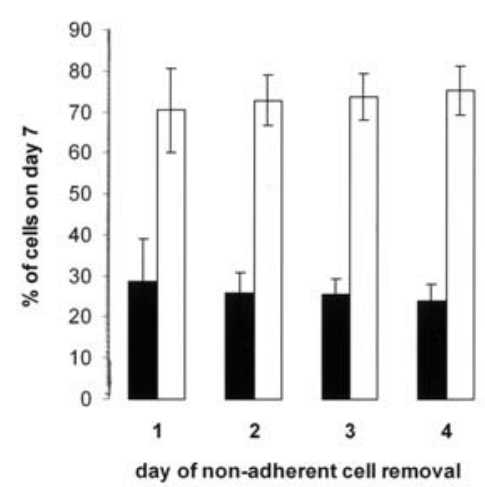

Quantification of CD45-positive cell contamination of primary MSC cultures. In order to quantitate the ratio between haematopoietic CD45-positive cells and MSCs, we employed threecolour flow cytometry (FACS) which allowed distinction between the two different cell types according to their surface antigens (CD45, CD29) and dead cells (Fig. 2A). Fig. 2B

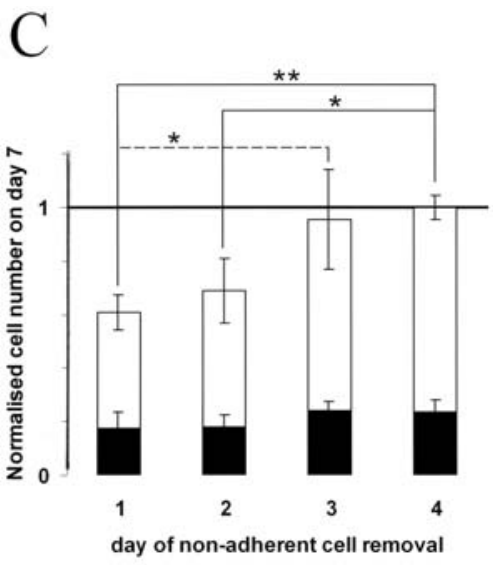

Figure 2. FACS analysis of bone marrow-derived stem cells prepared by adherence to plastic. (A) Representative dot-plot (out of 3 experiments) of rat MSCs isolated by adherence to plastic after 7 days of cell culture. The lower right quadrant contains CD29-highly positive and CD45-depleted MSCs while the upper right quadrant contains $\mathrm{HCs}$ with a CD45-positive and CD29-low expression profile. (B) Proportion of CD45-positive (black bars) and CD45-depleted (white bars) cells from rat MSCs isolated by adherence to plastic in relation to the time of the first medium change and therefore, removal of non-adherent cells. (C) Total yield of CD45-depleted (white bars) and CD45-positive (black bars) bone marrow-derived cells after 7 days of cell culture ( $\left.\mathrm{p}<0.05 ;{ }^{* *} \mathrm{p}<0.01\right)$. Yield of cells is normalized to cell number achieved when the non-adherent cells were removed on day 4 .

shows that the fraction of CD45-positive haematopoietic cells was not influenced by the adherence time after cell isolation. We mostly found $\sim 20-30 \%$ of the attached cells in bone marrow cell cultures to be CD45-positive when selected according to sole adherence to plastic surfaces. Flow cytometric results were concurrent with the observations made after 
A.

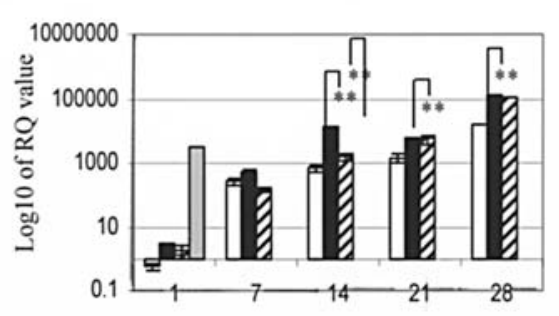

B.
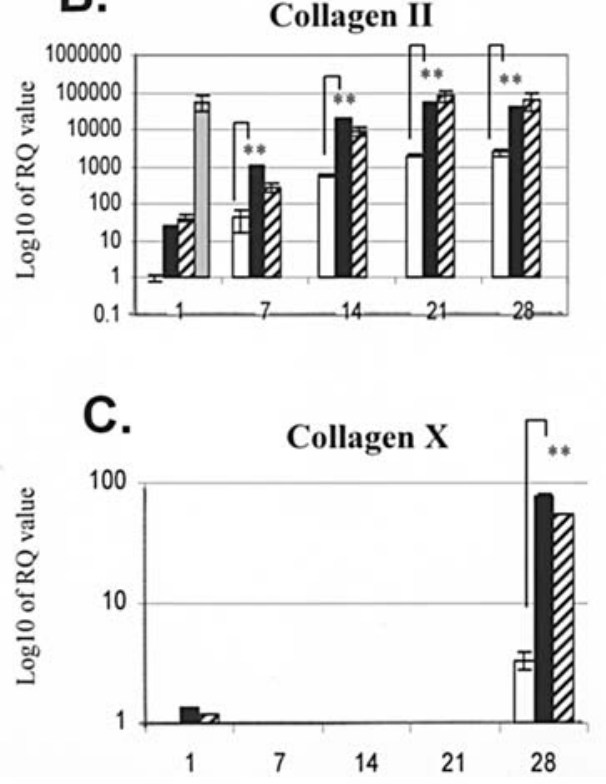

G.

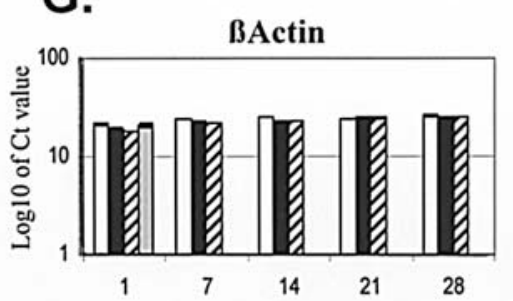

$\square$ CD45- depleted cells $\quad \square$ Adherent cells
$\square$ Remixed control cells $\quad \square$ Chondrocytes

$* P \leq 0.01 ; \cdot P \leq 0.05$
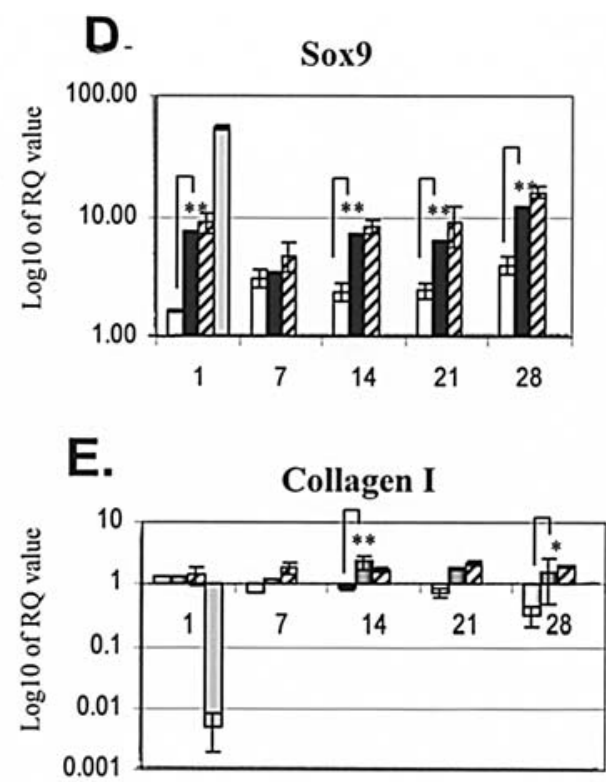

F.

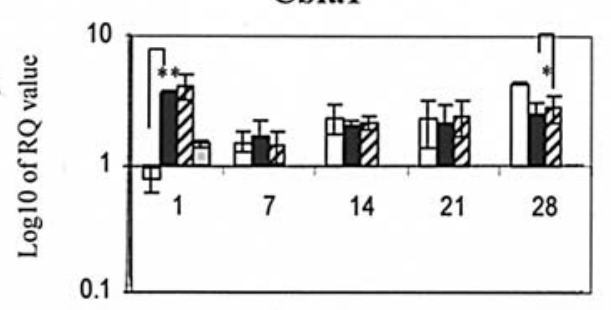

Figure 3. Chondrogenic gene expression profile of adherent MSCs, CD45depleted MSCs and remixed control cells. Relative quantitative mRNA expression level of chondrogenesis specific genes as COMP (A) Col2al (B) and Colloal (C), Sox9 (D), Collal (E), and Cbfal (F) was determined by employing the $\triangle \triangle C T$ method using SYBR I Green dye. Expression level of housekeeping gene, $B$-actin was determined as a controlling indicator of cell viability $(\mathrm{G})$ and is expressed as positive logarithm of CT-values. Expression level of each gene was compared between adherent, CD45-depleted and remixed control cells. Grey shaded bars indicate relative gene expression level of freshly isolated hip articular chondrocytes. Number of experiments each $=3$.

relative mRNA levels of COMP and collagen II, while Sox9 mRNA levels exhibited a moderate upregulation (Fig. 3A, B, and $\mathrm{D})$. Collagen $\mathrm{X}$ gene expression remained undetectable the first three weeks of cell culture; however, at day 28 expression of this collagen was found to be induced (Fig. 3C). The internal control gene, $B$-actin, remained unaltered during the complete time course of cell culturing (Fig. 3G). Comparing gene expression of CD45-depleted cells and the non-sorted population, we found collagen I gene expression to be increased in the non-sorted cell population during the culture period, while it became decreased in CD45-depleted MSCs. However, in CD45-depleted cells collagen I was still expressed at a relative higher level as in freshly isolated hip articular chondrocytes (Fig. 3E). Notably, the non-sorted cells displayed 
strikingly higher upregulation of collagen II, COMP and Sox9 gene expression compared to the CD45-depleted MSCs throughout the culture period. In the non-sorted population collagen II gene expression is upregulated on average 25 -fold, COMP gene expression $\sim 10$-fold, collagen $\mathrm{X}$ expression 23-fold and Sox9 mRNA levels 3-fold when compared to CD45-depleted MSCs. For collagen I gene expression we observed a significant difference only between the two groups at day 14 and 28, where the adherent MSCs demonstrated an upregulation resulting in a 3-5-fold higher gene expression when compared to CD45-depleted MSCs. Collagen I remained upregulated from day 14 on in adherent cells while CD45-depleted MSCs exhibit an increasingly downregulation during the time course of cell culture. We also observed a striking difference in the collagen $\mathrm{X}$ expression pattern between non-sorted and CD45-depleted cells. The collagen X mRNA expression profile of CD45-depleted cells experienced only a moderate increase of $\sim 3.3$-fold at the end of the culture period while adherent cells upregulated their mRNA level 76-fold compared to day 0 (Fig. 3C). Notably, Cbfa1 gene expression is differentially regulated with respect to the two cell populations (Fig. 3F). On day 1 Cbfal mRNA level is significantly higher in adherent cells (4.7-fold) and also in chondrocytes (2.0-fold) compared to CD45-depleted cells. This pattern became inverted at day 28 with CD45-depleted cells exhibiting an elevated expression (1.7-fold) compared to the adherent MSCs. In between these time points, relative gene expression of Cbfa1 is similarly regulated in both cell populations.

In remixed control cell populations (30\% CD45-positive cells with 70\% CD45-depleted cells) collagen II, X, I, Sox9 and Cbfa1 gene expression profile resembles closely that of adherent cells (Fig. 3B-F) and exhibits no significant differences. Only COMP gene expression at day 14 of the culture period significantly differs from adherent cells but the level at day 21 and 28 shows no difference to non-sorted cells (Fig. 3A). B-actin gene expression profile is similar for all three culture conditions (Fig. 3G).

\section{Discussion}

Adult bone marrow is the major source of haematopoietic and mesenchymal stem cells (1). In animal studies, adherence to tissue culture plastic is accepted as a satisfactory parameter for attaining marrow stromal cell population depleted of haematopoietic cells. However, when employing FACS analysis for surface antigen characterization we observed a contamination of these non-sorted MSCs with 20-30\% of CD45-positive cells. For additional characterization of sorted and non-sorted cell population we applied immunofluorescence staining for CD68 and D7Fib. The D7Fib antigen constitutes a fibroblast-specific molecule of yet unknown function, supposedly a specific marker for MSCs as suitable as STRO-1 and CD105 (20). We observed expression of the D7Fib antigen on adherent CD45-positive cells; however, these cells displayed a rounded morphology compared to the fibroblast-like CD45-depleted cells. CD68 and its murine analogue macrosialin, a member of the lamp family, appear to have a rather limited tissue distribution, being found on macrophages, Langerhans cells and dendritic cells (21). Notably, we have found all adherent cells CD68-positive suggesting that in non-committed undifferentiated cells, this protein might exhibit a different distribution pattern and possibly exert functions related to cell-cell or cell-ligand interactions (22).

CD45-positive haematopoietic cells were always detected in MSC cultures, regardless of the time of the first change of culture medium. This indicated a persistent presence and attachment of CD45-positive cells in the MSC environment when no further selection based on specific surface antigens is carried out. Adherence to the tissue culture plastic alone is a weak discriminating criterion for a homogeneous MSC population because neural cells, monocytes and macrophages are also known to be isolated according to their adherence to plastic surfaces $(6,23,24)$. The presence of CD45-positive haematopoietic cells and the variation of their proportion in primary cultures of MSCs could explain the occurrence of extensive variations usually seen in differentiation experiments with bone marrow-derived cells.

The fact that CD45-depleted cells expressed CD29 stronger than CD45-positive cells offered us an easier separation of two populations and limited the possible existence of more than two major subpopulations in our cultures. The integrin $B 1$ subunit CD29 exhibits a broad tissue distribution, including lymphocytes, endothelia, smooth muscle, and epithelia (25) and plays an important role in cellular processes, e.g. embryogenesis and HSC development. Although we did not find the haematopoietic cell fraction to vary according to the time of removal of non-adherent cells, we observed a correlation between adhesion time and yield of CD45-depleted marrow stromal cells. In parallel, cell adhesion plus proliferation could also be promoted by the prolonged presence of the non-adherent fraction via cytokine release and/or cell-cell contacts. A comparable effect of haematopoietic cells on MSC proliferation has been observed for megakaryocytes and platelets (26). Based on our results, we decided to leave the cells to adhere for 3 days before performing differentiation experiments.

Differentiation studies of HSCs have demonstrated that the small number of adherent MSCs present in total bone marrow aspirates provide an important microenvironment for growth of HSCs and their differentiation into several blood borne cell types. In long-term cultures of HSCs, these adherent cells even interact directly with the haematopoietic precursors (5). Herein, we showed that vice versa adherent CD45-positive haematopoietic precursor cells create a microenvironment which may enhance expression of particular marker genes in MSCs. For studying the effects of contaminating CD45-positive cells, we prepared a relatively homogeneous population of CD45-depleted MSCs by immunogenic depletion of CD45-positive cells. Both, the heterogenic adherent cells and the CD45-depleted subpopulation, were subjected to chondrogenic favourable 3-D environment by formation of a cell alginate amalgam. For control purposes in order to assure that difference in gene expression profile between sorted and unsorted MSCs is due to depletion of CD45-positive cells and not because of general depletion of differentiation competent cells or due to cell damage caused by the sorting process, we prepared a third population of remixed cells (30\% CD45-positive mixed with 70\% CD45depleted cells) following MACS preparation which was treated 
according to the differentiation protocol used for adherent and sorted cells.

We monitored relative gene expression of collagen II $(27)$ and COMP $(28,29)$ as examples for structural extracellular matrix proteins highly specific for hyaline cartilage. Additionally, we have investigated Sox 9 as a master transcription factor imperative for chondrogenic differentiation (13) and collagen I as differentiation control for a gene normally not active in healthy hyaline cartilage (30) but occurring together with collagen II in fibrocartilage (31). Gene expression of collagen $\mathrm{X}$, specific for terminal differentiated hypertrophic chondrocytes in the calcifying zone of cartilage and secondary ossification centres within the growth plate (32) indicates maturation of MSCs towards an undesired cartilage condition.

Gene expression of Cbfa1, a transcriptional activator for osteoblastic differentiation during the process of endochondral ossification is analyzed for monitoring differentiation towards osteogenic condition. Cbfa1 is also expressed in the pre-condensation stage during early development in a cell type with the potential to become either a chondrocyte or an osteoblast. In later developmental stages, expression of this transcription factor is restricted to prehypertrophic and hypertrophic chondrocytes where it acts as a hypertrophic differentiation inducer $(33,34)$.

The observed gene expression profile indicates not only a beneficial influence of CD45-positive cells on the differentiation process of MSCs towards the chondrogenic lineage, but also shows that when culture time is extended beyond 28 days chondrogenically differentiating MSCs might enter the prehypertrophic/hypertrophic differentiation cascade and proceed towards terminal differentiation. Usage of chondrogenic culture medium does not prevent upregulation of Cbfa1 and collagen $X$. This indicates either a not yet defined influence of medium components (i.e. TGF- $\beta$ or dexamethasone) on intrinsic cell-derived factors (i.e. signalling molecules, proteases, transcription factors) which are involved in regulation of the chondrogenic differentiation cascade or a medium independent process occurring by default through paracrine interaction of MSCs. A variety of growth factors i.e. members of the TGF- $\beta$ superfamily, IGF- 1 and bFGF could account for gene modulating effects in our system (35-38).

In conclusion, we demonstrated that bone marrow-derived fibroblast-like CD45-depleted rat MSCs share a heterogeneous cellular environment with $20-30 \%$ CD45-positive HCs, if no other selection procedure than adherence to plastic is employed. Our differentiation results indicate that adherent CD45-positive haematopoietic cells may create a microenvironment promoting differentiation of MSCs towards the chondrogenic lineage by upregulating specific genes. However, expression of undesired genes such as collagen I and collagen $\mathrm{X}$ is likewise promoted by HCs. On the contrary, at early condensation stage HCs strongly increase gene expression of Cbfa1 thus supporting chondrogenic differentiation of MSCs, while at a later differentiation stage when Cbfa1 plays an integral role in hypertrophy the regulation appears to be independent of the presence of HCs.

We speculate that the stromal CD45-positive population in toto is responsible for regulation of gene expression. The heterotrophic nature of bone marrow-derived cells suggest that in vivo interactions of different cell types is very likely and should be addressed accordingly. Characterization of additional subpopulations of CD45-positive cells according to their surface antigens and determination of their potential influence on gene expression may elaborate this point even further. Studies using conditioned media are needed to clarify whether physical contact between cells or paracrine factors, alone or in concurrence, are necessary for promoting gene expression leading to chondrogenic differentiation and maturation of MSCs. Identification and isolation of factors which are capable of modulating genes involved in differentiation and dedifferentiation processes of chondrocytes is vital for in vitro engineering of cartilage tissue repair.

\section{Acknowledgements}

We want to thank Maren Marschner for her excellent technical assistance and support. We also acknowledge Thomas Vogel for his generous help and scientific discussion. Special thanks go to Professor Achim Göpferich for his advice and support. The study was partly funded by the Bavarian Research Foundation (Bayerische Forschungsstiftung).

\section{References}

1. Pittenger MF, Mackay AM, Beck SC, Jaiswal RK, Douglas R, Mosca JD, et al: Multilineage potential of adult human mesenchymal stem cells. Science 284: 143-147, 1999.

2. Cancedda R, Dozin B, Giannoni P and Quarto R: Tissue engineering and cell therapy of cartilage and bone. Matrix Biol 22: 81-91, 2003

3. Bianco P, Riminucci M, Gronthos S and Robey PG: Bone marrow stromal stem cells: nature, biology, and potential applications. Stem Cells 19: 180-192, 2001

4. Galmiche MC, Koteliansky VE, Briere J, Herve P and Charbord P: Stromal cells from human long-term marrow cultures are mesenchymal cells that differentiate following a vascular smooth muscle differentiation pathway. Blood 82: 66-76, 1993.

5. Prockop DJ: Marrow stromal cells as stem cells for nonhematopoietic tissues. Science 276: 71-74, 1997.

6. Majumdar MK, Thiede MA, Mosca JD, Moorman M and Gerson SL: Phenotypic and functional comparison of cultures of marrow-derived mesenchymal stem cells (MSCs) and stromal cells. J Cell Physiol 176: 57-66, 1998.

7. Friedenstein AJ, Gorskaja JF and Kulagina NN: Fibroblast precursors in normal and irradiated mouse hematopoietic organs. Exp Hematol 4: 267-274, 1976.

8. Caplan AI and Bruder SP: Mesenchymal stem cells: building blocks for molecular medicine in the 21 st century. Trends Mol Med 7: 259-264, 2001.

9. Phinney DG, Kopen G, Isaacson RL and Prockop DJ: Plastic adherent stromal cells from the bone marrow of commonly used strains of inbred mice: variations in yield, growth, and differentiation. J Cell Biochem 72: 570-585, 1999.

10. Herbertson A and Aubin JE: Cell sorting enriches osteogenic populations in rat bone marrow stromal cell cultures. Bone 21: 491-500, 1997.

11. von der Mark: Structure and biosynthesis of collagens. In: Bone and Cartilage Metabolism. Seibel MJ, Robins SP and Bilezikian JP (eds). 1st edition, Academic press, pp3-29, 1999.

12. Bi W, Deng JM, Zhang Z, Behringer RR and De Crombrugghe B: Sox9 is required for cartilage formation. Nat Genet 22: 85-89, 1999.

13. De Crombrugghe B, Lefebvre V, Behringer RR, Murakami S, Bi W and Huang W: Transcriptional mechanisms of chondrocyte differentiation. Matrix Biol 19: 389-394, 2000.

14. Mundlos S and Olsen BR: Heritable diseases of the skeleton. Part I: Molecular insights into skeletal development transcription factors and signaling pathways. FASEB J 11: 125$132,1997$. 
15. Lefebvre V, Behringer RR and De Crombrugghe B: L-Sox5, Sox6 and Sox 9 control essential steps of the chondrocyte differentiation pathway. Osteoarthritis Cartilage 9 (Suppl A): S69-S75, 2001.

16. Dobson KR, Reading L, Haberey M, Marine X and Scutt A: Centrifugal isolation of bone marrow from bone: an improved method for the recovery and quantitation of bone marrow osteoprogenitor cells from rat tibiae and femurae. Calcif Tissue Int 65: 411-413, 1999

17. Maniatopoulos C, Sodek J and Melcher AH: Bone formation in vitro by stromal cells obtained from bone marrow of young adult rats. Cell Tissue Res 254: 317-330, 1988.

18. Johnstone B, Hering TM, Caplan AI, Goldberg VM and Yoo JU: In vitro chondrogenesis of bone marrow-derived mesenchymal progenitor cells. Exp Cell Res 238: 265-272, 1998.

19. Ginzinger DG: Gene quantification using real-time quantitative PCR: an emerging technology hits the mainstream. Exp Hematol 30: 503-512, 2002.

20. Jones EA, Kinsey SE, English A, Jones RA, Straszynski L, Meredith DM, et al: Isolation and characterization of bone marrow multipotential mesenchymal progenitor cells. Arthritis Rheum 46: 3349-3360, 2002.

21. Ramprasad MP, Terpstra V, Kondratenko N, Quehenberger O and Steinberg D: Cell surface expression of mouse macrosialin and human CD68 and their role as macrophage receptors for oxidized low density lipoprotein. Proc Natl Acad Sci USA 93: 14833-14838, 1996.

22. Fukuda M: Lysosomal membrane glycoproteins. Structure, biosynthesis, and intracellular trafficking. J Biol Chem 266: 21327-21330, 1991.

23. Delles AM, Rittenhouse-Olson K, Morgan J and Oseroff AR: A simple method for the purification of human peripheral blood antigen presenting cells (dendritic cells, monocytes/macrophages, and B lymphocytes). Immunol Invest 31: 233-245, 2002.

24. Kiefer F, Wagner EF and Keller G: Fractionation of mouse bone marrow by adherence separates primitive hematopoietic stem cells from in vitro colony-forming cells and spleen colony-forming cells. Blood 78: 2577-2582, 1991.

25. Springer TA: Adhesion receptors of the immune system. Nature 346: 425-434, 1990

26. Castro-Malaspina H, Rabellino EM, Yen A, Nachman RL and Moore MA: Human megakaryocyte stimulation of proliferation of bone marrow fibroblasts. Blood 57: 781-787, 1981.

27. Mendler M, Eich-Bender SG, Vaughan L, Winterhalter KH and Bruckner P: Cartilage contains mixed fibrils of collagen types II, IX, and XI. J Cell Biol 108: 191-197, 1989.
28. Hedbom E, Antonsson P, Hjerpe A, Aeschlimann D, Paulsson M, Rosa-Pimentel E, et al: Cartilage matrix proteins. An acidic oligomeric protein (COMP) detected only in cartilage. J Biol Chem 267: 6132-6136, 1992.

29. Di Cesare P, Hauser N, Lehman D, Pasumarti S and Paulsson M: Cartilage oligomeric matrix protein (COMP) is an abundant component of tendon. FEBS Lett 354: 237-240, 1994.

30. Rossert J, Terraz C and Dupont S: Regulation of type I collagen genes expression. Nephrol Dial Transplant 15 (Suppl 6): 66-68, 2000.

31. Nerlich AG, Boos N, Wiest I and Aebi M: Immunolocalization of major interstitial collagen types in human lumbar intervertebral discs of various ages. Virchows Arch 432: 67-76, 1998.

32. Schmid TM, Popp RG and Linsenmayer TF: Hypertrophic cartilage matrix. Type X collagen, supramolecular assembly, and calcification. Ann NY Acad Sci 580: 64-73, 1990.

33. Karsenty G: Minireview: transcriptional control of osteoblast differentiation. Endocrinology 142: 2731-2733, 2001.

34. Takeda S, Bonnamy JP, Owen MJ, Ducy P and Karsenty G: Continuous expression of $\mathrm{Cbfa} 1$ in nonhypertrophic chondrocytes uncovers its ability to induce hypertrophic chondrocyte differentiation and partially rescues Cbfa1-deficient mice. Genes Dev 15: 467-481, 2001.

35. Bohme K, Winterhalter KH and Bruckner P: Terminal differentiation of chondrocytes in culture is a spontaneous process and is arrested by transforming growth factor-beta 2 and basic fibroblast growth factor in synergy. Exp Cell Res 216: 191-198, 1995.

36. Recklies AD, Baillargeon L and White C: Regulation of cartilage oligomeric matrix protein synthesis in human synovial cells and articular chondrocytes. Arthritis Rheum 41: 997-1006, 1998.

37. Murakami S, Kan M, McKeehan WL and De Crombrugghe B: Up-regulation of the chondrogenic Sox 9 gene by fibroblast growth factors is mediated by the mitogen-activated protein kinase pathway. Proc Natl Acad Sci USA 97: 1113-1118, 2000.

38. Horton WE Jr, Higginbotham JD and Chandrasekhar S: Transforming growth factor-beta and fibroblast growth factor act synergistically to inhibit collagen II synthesis through a mechanism involving regulatory DNA sequences. J Cell Physiol 141: 8-15, 1989 . 